\title{
Is intra-articular infliximab therapy a good alternative to radionuclide synovectomy for a patient with refractory pigmented villonodular synovitis?
}

\author{
Anna Felis-Giemza ID , Anna Apanel-Kotarska ID, Marek Chojnowski ID, Marzena Olesińska ID \\ Department of Systemic Connective Tissue Diseases, National Institute of Geriatrics, Rheumatology, and Rehabilitation, Warsaw, Poland
}

\begin{abstract}
Pigmented villonodular synovitis (PVNS) is a rare disease that has clinical and histopathological characteristics of both benign proliferative disorder and a chronic inflammatory process of the synovial tissue. The primary mode of treatment is surgery followed by an adjuvant radiotherapy; however, the risk of recurrence is a significant (40-70\%). Several publications suggest that the TNF- $\alpha$ inhibitors might be a treatment option. We present a case of a 29 -year-old female diagnosed with PVNS of the knee joint, refractory to surgery and 3 radionuclide synovectomies. Because the possibilities of conventional therapy were exhausted, treatment with an intra-articular anti-TNF- $\alpha$ monoclonal antibody (infliximab) was performed. Despite a high safety profile and a good tolerance of that therapy we did not observe significant clinical and radiological improvement. To assess the effectiveness of intra-articular TNF- $\alpha$ inhibitors as an adjuvant treatment in PVNS, prospective studies are needed.
\end{abstract}

Key words: pigmented villonodular synovitis, TNF- $\alpha$ inhibitor, intra-articular therapy.

\section{Introduction}

Pigmented villonodular synovitis (PVNS), or diffuse-type tenosynovial giant cell tumour (D-TGCT), is a rare disease of unknown origin affecting synovial tissue, with locally destructive effects on articular and para-articular structures.

Pigmented villonodular synovitis usually affects adolescents and middle-aged people, with women twice as likely to be affected. Some studies suggest females are slightly more predisposed, but only in the term of localized disease [1, 2], however, others suggest that male and female genders are affected at equal rates [3].

The disease typically has a monoarticular course, involving the knee joint (75\%) or hip joint (15\%), and less commonly other joints (ankle, wrist, elbow, or shoulder joints) [4].

Histopathologically, PVNS is characterized by synoviocyte proliferation and inflammatory infiltrations of lipophages and macrophages loaded with hemosiderin. Macroscopically, the prominent feature of the disease is the formation of nodules and villi of the synovial membrane of joints, bursae, and tendon sheaths.

Although histologically benign, these villonodular lesions can potentially infiltrate cartilage, bone and extraarticular structures, leading to functional impairment, disability, and, in extreme cases, necessity of limb amputation.

The primary mode of treatment is a surgical resection of the synovial tissue, either arthroscopically or by means of open, multi-compartment synovectomy [5]. The chances of long-term remission are increased when the surgery is followed with an adjuvant radiotherapy such as radionuclide synovectomy (RSV) or external beam radiotherapy (EBRT) [6]. Despite multi-modality treatment, the risk of recurrence is significant, ranging between 40 and $70 \%$ [7].

So far, there are no universally accepted systemic therapies that are effective in PVNS. Several publications suggest the tumour necrosis factor $\alpha$ (TNF- $\alpha$ ) inhibitors might play a role in recurrent, treatment-refractory PVNS. Another, colony-stimulating factor 1 receptor (CSF-1R) and its ligands,

Address for correspondence:

Anna Felis-Giemza, Department of Systemic Connective Tissue Diseases, National Institute of Geriatrics, Rheumatology, and Rehabilitation, 1 Spartańska St., 02-637 Warszawa, Poland, e-mail: annafelis@wp.pl

Submitted: 01.07.2021; Accepted: 01.10.2021 
CSF-1, regulate the function and survival of tumour-associated macrophages, which are involved in tumourigenesis and in the suppression of antitumour immunity. The colony-stimulating factor 1 receptor/CSF-1 axis has been implicated in the pathogenesis of pigmented villonodular synovitis (PVNS), a benign tumour of the synovium.

\section{Material and methods}

There are few data in the literature on the intra-articular treatment of PVNS. There are some reports of surgical treatment followed by an adjuvant radiotherapy $[5,6]$. The relevant literature describing intra-articular treatment PVNS with TNF alfa inhibitors was reviewed.

A systematic search of the literature on the electronic database PubMed was conducted using the following combination of words: pigmented villonodular synovitis, combined with TNF alfa inhibitors (infliximab, etanercept, adalimumab, golimumab, certolizumab pegol) and intra-articular and treatment - between January 2005 and December 2020.

From the review of available literature in the last 15 years, only those directly describing TNF alfa inhibitor intra-articular treatment of PVNS were taken into account. Studies were also included if they evaluated not only surgical treatment but also RSV and either small molecules or antibodies in adult patients with PVNS. Articles describing PVNS treatment of children were not included.

The language of the chosen articles was restricted to English. The discussion was based on the case study and a literature review (Fig. 1).

\section{Case description}

We present a case of a 29-year-old Caucasian female with histopathologically confirmed PVNS of the right knee joint. She underwent open surgical synovectomy followed with RSV (400 MBq of 90-yttrium citrate) with good initial results (Fig. 2).

One year after the first-line treatment, the symptoms recurred, and the presence of villonodular lesions in the right knee joint was confirmed with ultrasound (US) and magnetic resonance imaging (MRI). The second RSV with 90-yttrium citrate was performed, inducing remission for another 15 months. After the third RSV, the treatment effects lasted only 6 months. At that point, another RSV was not recommended, and the patient did not consent to undergo surgical intervention. Since the possibilities of standard therapies were exhausted, an attempt of experimental treatment with intra-articular TNF- $\alpha$ inhibitor was made.

According to singular case-studies, the most common and safest TNF- $\alpha$ inhibitor used to treat PVNS was infliximab $[8,9]$, which made it the drug of choice for our patient.
After obtaining approval of the Ethics Committee and written consent of the patient, the standard diagnostics as before any other biological treatment was performed, i.e. complete blood count, biochemical markers of liver and kidney function, human immune deficiency and hepatitis B/C virus assays, chest plain radiograph, breast US, and pulmonological and gynaecological consultation.

All the results were within normal limits, no contraindications for biological treatment were found. Right knee MRI was also performed, which showed pathological synovial hypertrophy and a fluid collection in the suprapatellar recess (Fig. 3). The infliximab (Remsima) was obtained as a donation from EGIS Polska.

The patient was administered a total of 7 infliximab injections, according to the 0-1-3-6-12-15-month regimen. Each time, the right knee joint was punctured using an aseptic technique under direct ultrasonographic guidance and $100 \mathrm{mg}$ of infliximab dissolved in $10 \mathrm{ml}$ of physiological saline was injected. Three months after the last injection, a knee MRI was performed to assess the treatment effects.

\section{Results}

Throughout the treatment period and 3 months after last infliximab injection, no significant clinical improvement was observed. Oedema, tenderness, and restriction of motion of the knee joint persisted. During each of the joint punctures, the excess of pinkish synovial fluid was evacuated.

The final MRI revealed disease progression, with generalized synovial hypertrophy, effusion, and numerous loose bodies in the joint cavity. The patient was subsequently referred for surgical synovectomy and joint debridement.

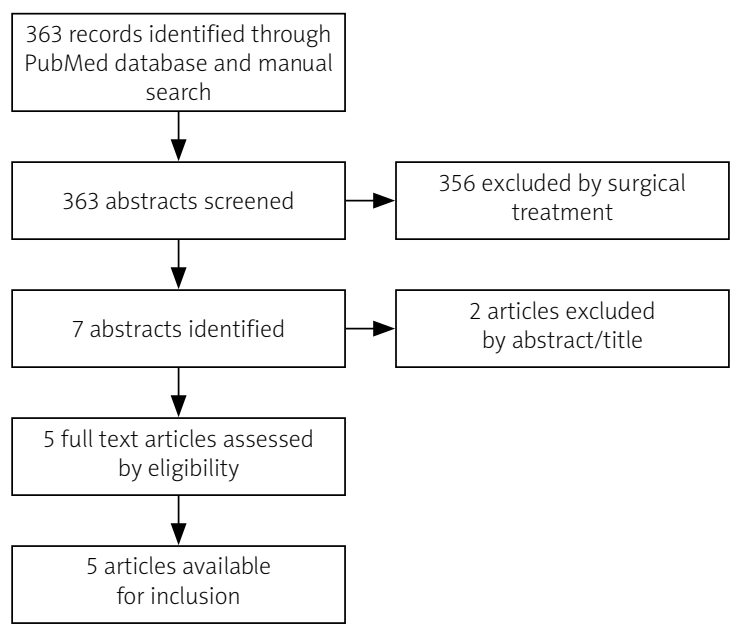

Fig. 1. Literature screening algorithm for articles included in the systematic review. 

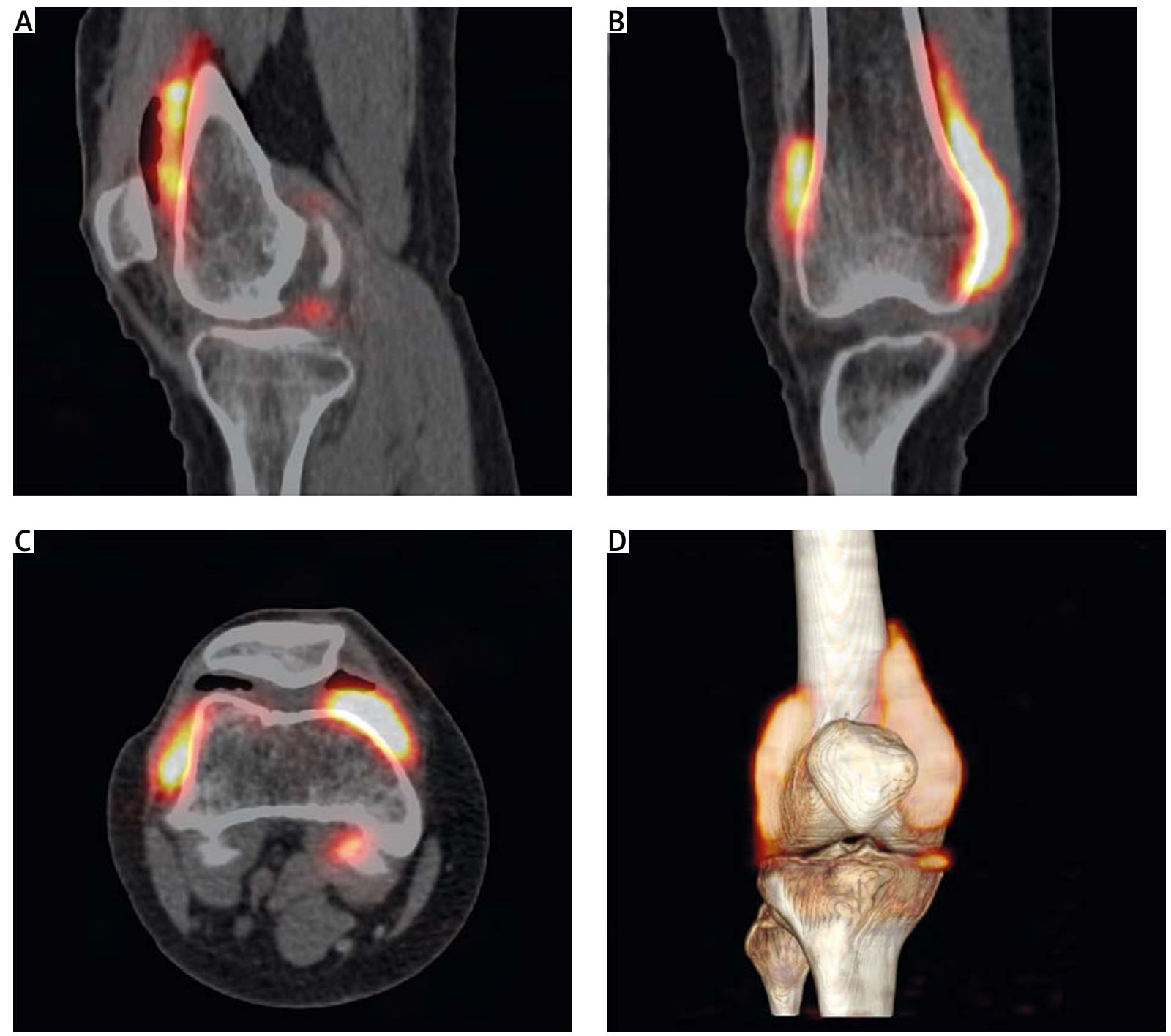

Fig. 2. Positron emission tomography/computed tomography with ${ }^{90}$-citrate after radiosynovectomy of the right knee joint (sagittal, coronal and transverse cross-sections, and a 3D volume rendering). Diffuse distribution of radiopharmaceutical in all compartments of the joint.

The patient tolerated the treatment with infliximab very well. There were no adverse effects; of particular importance, there were no signs of septic arthritis or systemic infection.

\section{Discussion}

Pigmented villonodular synovitis has clinical and histopathological characteristics of both benign proliferative disorder and a chronic inflammatory process. In essence, it involves a polyclonal proliferation of activated synovial macrophages, which, through secretion of TNF- $\alpha$ and other cytokines, continuously sustain inflammation in the synovial membrane and stimulate its invasive growth.
Similarly to systemic inflammatory arthropathies, inhibition of TNF- $\alpha$ in PVNS potentially leads to a reduction of the synovial inflammation and resolution of clinical symptoms. However, the gold standard of treatment for pigmented villonodular synovitis has traditionally been surgical excision with total synovectomy of the affected joint, either with an open or arthroscopic approach [10].

While both methods have a similar reoccurrence rate of PVNS post-surgery, the arthroscopic procedure has consistently demonstrated better functional and range of motion outcomes for the patient $[5,10,11]$.

Recent literature reports single case studies demonstrating good effects of PVNS treatment with intra-articular adalimumab, etanercept, and infliximab. Moreover, the analysis of 1046 intra-articular injections of various 


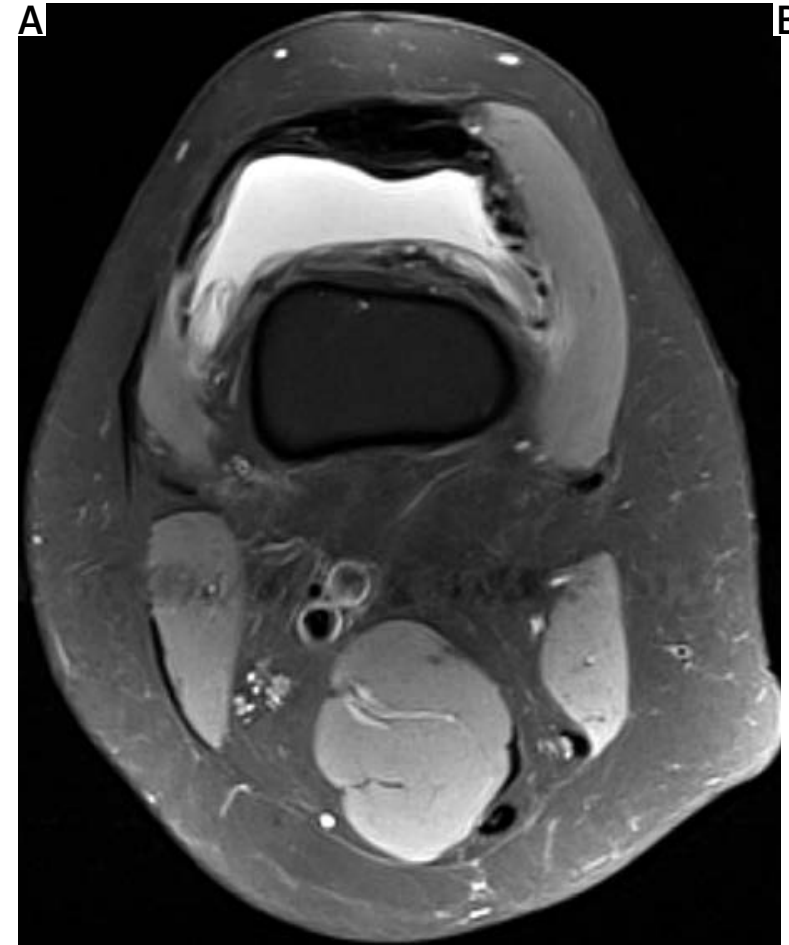

TNF- $\alpha$ inhibitors suggests a very high safety profile of such therapy [12].

Colony-stimulating factor 1 receptor (CSF-1R) and its ligands, CSF-1 and interleukin 34 (IL-34), regulate the function and survival of tumour-associated macrophages, which are involved in tumourigenesis and in the suppression of antitumour immunity. Targeting CSF-1R via either small molecules or antibodies has shown interesting results in vitro but limited antitumor activity in vivo, in solid tumours.

Concerning PVNS, clinical trials assessing CSF-1R inhibitors have revealed promising initial outcomes. Blocking CSF-1/CSF-1R signalling represents a promis-

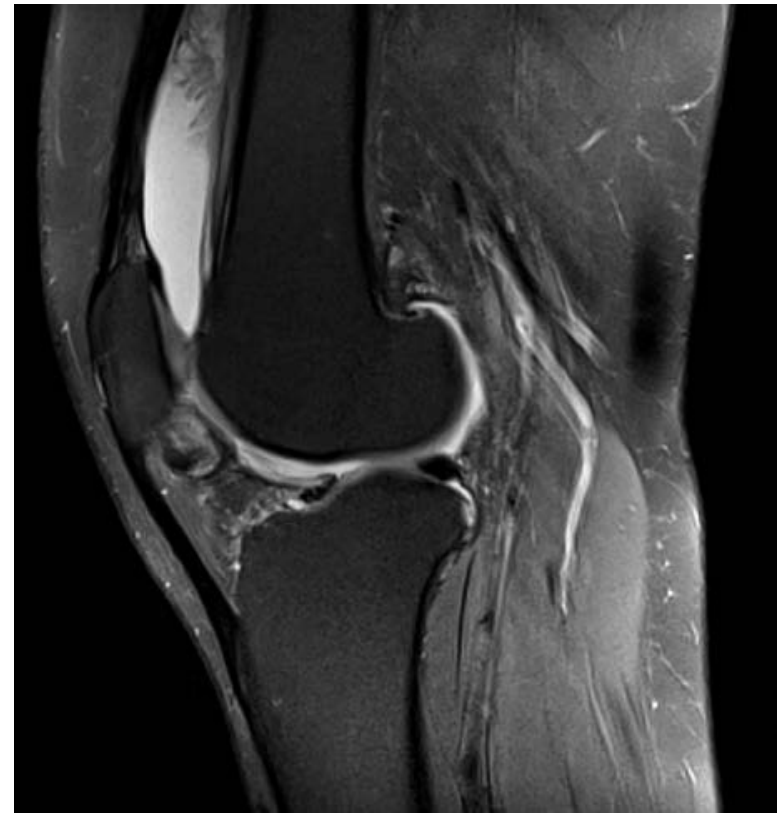

Fig. 3. T2-wieghted fast spin-echo magnetic resonance imaging of the right knee joint. Effusion and synovial villi in the suprapatellar recess.

ing immunotherapy approach and several new potential combination therapies for future clinical testing [13].

Research from the years 2005-2020 includes 8 case studies of PVNS patients treated with systemic or intra-articular TNF- $\alpha$ inhibitors (Table I).

Kroot et al. [8] reported a case of a patient showing a significant clinical improvement after intra-articular infliximab, along with a histopathologically confirmed decreased expression of TNF- $\alpha$ in the synovial membrane of the affected joint.

Fiocco at al. [14] described 2 patients with recurrent PVNS, successfully treated with intra-articular etanercept injections. The patients regained full range of mo-

Table I. Intra-articular therapy with tumour necrosis factor- $\alpha$ antagonists for severe diffuse pigmented villonodular synovitis: drug, dosing, and clinical efficacy

\begin{tabular}{|c|c|c|}
\hline $\begin{array}{l}\text { Intra-articular therapy } \\
\text { (No. of cases) }\end{array}$ & Study & Dosing and effect of treatment (if data available) \\
\hline Infliximab (1) & Kroot et al., 2005 ] & $\begin{array}{c}\text { INF dose: } 5 \mathrm{mg} / \mathrm{kg} \text { and the time between injections was } \\
\text { no longer than } 8 \text { weeks (2, 6, 14, and } 20 \text { weeks later, } \\
\text { and bimonthly thereafter up to } 54 \text { weeks); controlled } \\
\text { the signs and symptoms }\end{array}$ \\
\hline $\begin{array}{l}\text { Etanercept (2) } \\
\text { Etanercept (1) }\end{array}$ & $\begin{array}{l}\text { Fiocco et al., } 2006[14] \\
\text { Zhao X. et al., } 2014[19]\end{array}$ & $\begin{array}{c}12.5 \text { mg weekly IA-ETN injection for } 4 \text { weeks, followed by } \\
\text { extended arthroscopic synovectomy and } 25 \text { mg IA-ETN } \\
\text { injection for } 4 \text { weeks; marked remission } \\
\text { IA-ETN injection; 1-year remission }\end{array}$ \\
\hline Adalimumab (1) & Kobak, $2011[15]$ & Significant clinical and radiological disease regression \\
\hline Infliximab (3) & Praino et al., 2015 [9] & $\begin{array}{c}6 \text { injections of } 100 \text { mg infliximab within } 12 \text { months; } \\
\text { complete remission }\end{array}$ \\
\hline
\end{tabular}

IA-ETN-intra-articular etanercept. 
tion in the treated joints, and US evaluation revealed reduction of thickness of the synovial tissue.

Similarly, Kobak [15] reported clinical and radiological improvement after intra-articular adalimumab in a patient who did not consent to surgical intervention.

Praino et al. [9] effectively treated 3 patients suffering from severe, refractory PVNS with intra-articular infliximab. During a 12-month observation, all the patients remained symptom-free, with decrease of synovial hypertrophy and joint effusion in imaging studies. No adverse effects of the treatment were observed.

In our case, we did not observe a satisfactory response to the treatment. In imaging studies there was marked disease progression. The unfavourable results might be attributed to inadequate dosing and the long intervals between injections. In the study by Kroot et al. [8], the infliximab dose was $5 \mathrm{mg} / \mathrm{kg}$ and the time between injections was no longer than 8 weeks.

However, Praino et al. [9] reported good results with treatment regimen similar to ours, i.e. 6 injections of $100 \mathrm{mg}$ infliximab within 12 months.

To properly assess the effectiveness of intra-articular TNF- $\alpha$ inhibitors as an adjuvant treatment in recurrent PVNS, prospective studies are needed, with larger numbers of patients and longer observation periods.

Research from the years 2005-2020 also includes studies of PVNS patients treated with other monoclonal antibodies and tyrosine kinase inhibitors, considering a novel CSF-1 pathway.

Emactuzumab and PLX3397 deserve a mention [2]. Emactuzumab is a monoclonal antibody that binds directly to the CSF-1 receptor on the surface of macrophages, thereby reducing or eliminating the effects of inappropriate CSF-1 production.

In a recent phase I study, 26 of 28 patients with PVNS had an objective response to treatment with emactuzumab [2]. The CSF1R inhibitor PLX3397 (which acts by blocking the molecular endpoints of (SF-1) showed not only a well-tolerated side effect profile but also a clinical response in $52 \%$ of patients $[6,16]$.

Most recently, the use of pexidartinib, a CSF-1 receptor antagonist, was approved by the FDA in August 2019 for use in patients with extensive disease, who are unlikely to benefit from surgical intervention [17].

The continuous activation of macrophages in PVNS is attributed to the overexpression of the macrophage colony stimulating factor-1 (CSF1) due to a mutation in the CSF1 gene, and the resulting overstimulation of the CSF1 receptor (CSF1R). It was shown that this pathway can be non-specifically affected by tyrosine kinase inhibitors such as imatinib or nilotinib [18]. However, a more targeted therapy is possible [19].
Pexidartinib is a novel, recently registered small molecule and is the first systemic therapy to be approved for PVNS. While the initial results are encouraging, it is important to note that a phase III trial had an overall response rate of only 39\% [17].

Cassier PA et al. [20] described 29 patients from 12 institutions in Europe, Australia, and the United States treated with imatinib mesylate (IM - CSF1R inhibitor). Symptomatic improvement was noted in 16 of 22 patients (73\%) who were assessable for symptoms.

The authors concluded that the benefits of alleviating morbidity in patients with localized PVNS/TGCT must be balanced against the potential toxicity of chronic drug therapy.

\section{Conclusions}

The mainstay of current PVNS treatment is surgery with adjuvant radiotherapy. Despite combination therapy, the recurrence rate can be as high as 70\%, which is far from satisfactory. It highlights the need for a conservative therapy that could sustain remission after initial invasive treatment. Although promising, it remains to be determined whether TNF-ablockade can influence longterm outcomes in PVNS patients.

Another potential answer to the PVNS issue may be targeted, molecular therapy.

So far, in patients with recurrent and treatment refractory PVNS, approved therapeutic options are limited and the optimal standard of care remains to be determined.

The authors declare no conflict of interest.

\section{References}

1. Mastboom MJL, Verspoor FGM, Verschoor AJ, et al. Higher incidence rates than previously known in tenosynovial giant cell tumors. Acta Orthop 2017; 88: 688-694, DOI: 10.1080/17453674.2017.1361126.

2. Brahmi M, Vinceneux A, Cassier PA. Current systemic treatment options for tenosynovial giant cell tumor/pigmented villonodular synovitis: targeting the CSF1/CSF1R Axis. Curr Treat Options Oncol 2016; 17: 10, DOI: 10.1007/s11864-0150385-x.

3. West RB, Rubin BP, Miller MA, et al. A landscape effect in tenosynovial giant-cell tumor from activation of CSF1 expression by a translocation in a minority of tumor cells. Proc Natl Acad Sci U S A 2006; 103: 690-695, DOI: 10.1073/pnas.0507321103.

4. Greenspan A, Grainger AJ. Articular abnormalities that may mimic arthritis. J Ultrason 2018; 18: 212-223, DOI: 10.15557/ JoU.2018.0032.

5. Mollon B, Lee A, Busse JW, et al. The effect of surgical synovectomy and radiotherapy on the rate of recurrence of pig- 
mented villonodular synovitis of the knee: an individual patient meta-analysis. Bone Joint J 2015; 97-B: 550-557, DOI: 10.1302/0301-620X.97B4.34907.

6. Fang Y, Zhang Q. Recurrence of pigmented villonodular synovitis of the knee: a case report with review of literature on the risk factors causing recurrence. Medicine (Baltimore) 2020; 99: e19856, DOI: 10.1097/MD.0000000000019856.

7. Verspoor FG, Zee AA, Hannink G, et al. Long-term follow-up results of primary and recurrent pigmented villonodular synovitis. Rheumatology (Oxford) 2014; 53: 2063-2070, DOI: 10.1093/rheumatology/keu230.

8. Kroot EJ, Kraan MC, Smeets TJ, et al. Tumour necrosis factor alpha blockade in treatment resistant pigmented villonodular synovitis. Ann Rheum Dis 2005; 64: 497-499, DOI: 10.1136/ ard.2004.025692.

9. Praino E, Lapadula G, Scioscia C, et al. Refractory knee giant cell tumor of the synovial membrane treated with intra-articular injection of Infliximab: a case series and review of the literature. Int J Rheum Dis 2015; 18: 908-912, DOI: 10.1111/ 1756-185X.12585.

10. Chang JS, Higgins JP, Kosy JD, Theodoropoulos J. Systematic arthroscopic treatment of diffuse pigmented villonodular synovitis in the knee. Arthrosc Tech 2017; 6: e1547-e1551, DOI: 10.1016/j.eats.2017.06.029.

11. Yang B, Liu D, Lin J, et al. Surgical treatment of diffuse pigmented villonodular synovitis of the knee. Zhongguo Yi Xue Ke Xue Yuan Xue Bao 2015; 37: 234-239, DOI: 10.3881/j. issn.1000-503X.2015.02.017.

12. Bello S, Bonali C, Serafino L, et al. Intra-articular therapy with tumor necrosis factor- $\alpha$ antagonists: an update. Reumatismo 2014; 65: 257-263, DOI: 10.4081/reumatismo.2013.721.
13. Peyraud F, Cousin S, Italiano A. CSF-1R inhibitor development: current clinical status. Curr Oncol Rep 2017; 19: 70, DOI: 10.1007/s11912-017-0634-1.

14. Fiocco U, Sfriso P, Oliviero F, et al. [Intra-articular etanercept treatment for severe diffuse pigmented villonodular knee]. Reumatismo 2006; 58: 268-274 [Article in Italian].

15. Kobak S. Intraarticular adalimumab in a patient with pigmented villonodular synovitis. Rheumatol Int 2011; 31: 251-254, DOI: 10.1007/s00296-009-1185-z.

16. Fecek C, Carter KR. Pigmented villonodular synovitis. In: StatPearls [Internet]. Treasure Island (FL): Stat. Pearls Publishing; 2021.

17. Tap WD, Gelderblom H, Palmerini E, et al. Pexidartinib versus placebo for advanced tenosynovial giant cell tumour (ENLIVEN): a randomised phase 3 trial. Lancet 2019; 394: 478-487, DOI: 10.1016/S0140-6736(19)30764-0.

18. Verspoor FGM, Mastboom MJL, Hannink G, et al. Long-term efficacy of imatinib mesylate in patients with advanced Tenosynovial Giant Cell Tumor. Sci Rep 2019; 9: 14551, DOI: 10.1038/ s41598-019-51211-y.

19. Zhao X, Ji W, Qian X, Lu Y. Pigmented villonodular synovitis developing in a patient with rheumatoid arthritis. J Clin Rheumatol 2014; 20: 283-286, DOI: 10.1097/RHU.0000000000000119.

20. Cassier PA, Gelderblom H, Stacchiotti S, et al. Efficacy of imatinib mesylate for the treatment of locally advanced and/or metastatic tenosynovial giant cell tumor/pigmented villonodular synovitis. Cancer 2012; 118: 1649-1655, DOI: 10.1002/ cncr.26409. 